\title{
Preliminary Comparison Study of Two Electro-Mechan- ical Cardiopulmonary Resuscitation Devices
}

\author{
Alejandro Mendoza García, Stefan Eichhorn, Annemarie Stroh, Marcin \\ Polski, Alois Knoll \\ Technical University Munich \\ Munich, Germany
}

Introduction: Over the past years several electro-mechanical devices have become available to the market, enabling paramedics to transport patients with cardiogenic shock to hospitals, while maintaining constant perfusion. These devices however have various mechanical structures and compression mechanisms that can affect the body perfusion. The presented paper shows the preliminary results of the comparison of two of such CPR devices using a pig model: the Lucas 2 and the Corpuls CPR.

Methods: Two middle sized domestic pigs weighing $30+-5 \mathrm{~kg}$ were used for this study. They were properly pre-medicated and given general anesthesia.

As preparation several sensors were placed to obtain vital parameters and blood flow during CPR. A normal oximeter was placed in the ear, and two regional oximetry sensors were placed at the level of the neck and on the tongue. Additionally the pressure at the left ventricle was captured using a Millar tip. The ECG and CO2 were obtained using the Corpuls 3 monitoring system. After preparations were finished and before CPR all the parameters were recorded as a baseline. Afterwards the heart was stopped for 5 minutes and afterwards the CPR device was activated.

Results: The results obtained show that during the capturing of baseline parameters the mean carotid flow was of $0,31 \mathrm{~mL} / \mathrm{min}$ for the pig prepared for the Lucas 2 device and $230 \mathrm{~mL} / \mathrm{min}$ for the Corpuls device. After 3 minutes of CPR compressions the Lucas device generated $25 \%$ of the mean carotid flow, while the Corpuls device generated $76 \%$ of the carotid mean flow.
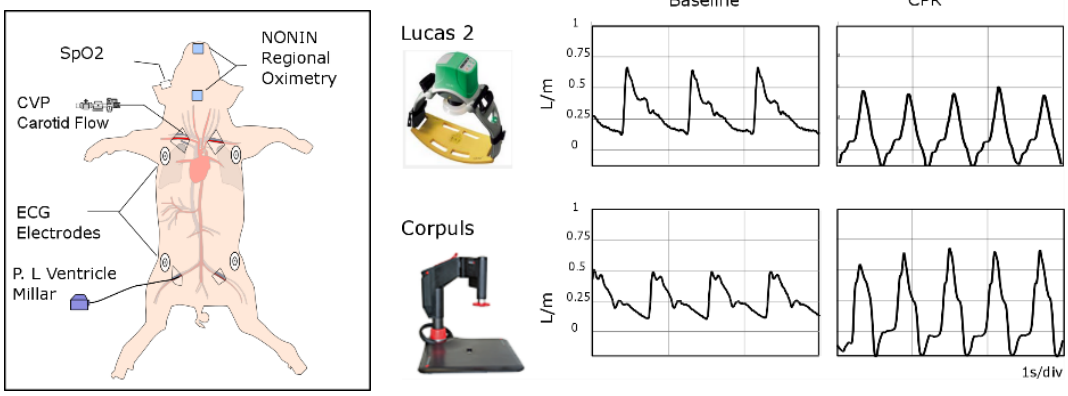